\title{
Transfection of neurotrophin-3 into neural stem cells using ultrasound with microbubbles to treat denervated muscle atrophy
}

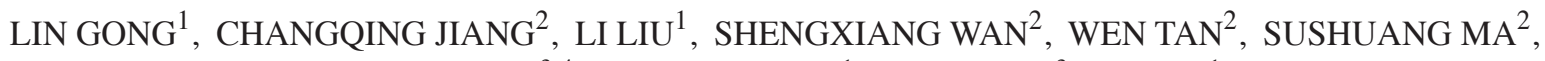 \\ XIAOJIAN JIA ${ }^{3,4}$, MEIWEI WANG ${ }^{1}$, AZHEN HU $^{3}$, YU SHI ${ }^{1}$, \\ YU ZHANG ${ }^{5}$, YUANYUAN SHEN ${ }^{6}$, FENG WANG ${ }^{3,4,7}$ and YUN CHEN ${ }^{1,3}$

\begin{abstract}
Departments of ${ }^{1}$ Ultrasound and ${ }^{2}$ Sports Medicine, Peking University Shenzhen Hospital, Shenzhen; ${ }^{3}$ Shenzhen Key Laboratory for Drug Addiction and Medication Safety, Biomedical Research Institute, Shenzhen Peking University, The Hong Kong University of Science and Technology Medical Center, Shenzhen, Guangdong 518036; ${ }^{4}$ Shenzhen Kangning Hospital \& Shenzhen Mental Health Center, Shenzhen, Guangdong 518020; ${ }^{5}$ Department of Ultrasound, The Third People's Hospital of Shenzhen, Shenzhen, Guangdong 518055; ${ }^{6}$ Department of Biomedical Engineering,

National Regional Key Technology Engineering Laboratory for Medical Ultrasound, Shenzhen

University, Shenzhen, Guangdong 518060; ${ }^{7}$ Department of Physiology and Neurobiology,

Xinxiang Medical University, Xinxiang, Henan 453002, P.R. China
\end{abstract}

Received February 15, 2017; Accepted July 27, 2017

DOI: $10.3892 /$ etm.2017.5439

\begin{abstract}
Neurotrophin-3 (NT-3) has potential as a therapeutic agent for the treatment of patients with denervated muscle atrophy. However, the endogenous secretion of NT-3 is low and exogenous NT-3 lacks sufficient time to accumulate due to its short half-life. The transfection of NT-3 has been demonstrated to have a beneficial effect on denervated muscle and motor endplates. Neural stem cells (NSCs) differentiate into neurons and form motor endplate nerve-muscle connections. It has been previously demonstrated that local and noninvasive transfection can be performed using ultrasound with microbubbles (MBs). In the current study, hematoxylin and eosin, acetylcholinesterase and gold chloride staining, as well as transmission electron microscopy, were performed to verify the effects of this treatment strategy. The results demonstrated that using ultrasound with MBs for the transfection of NT-3 into NSCs, and their subsequent transplantation in vivo, attenuated the atrophy of denervated muscle and reduced motor
\end{abstract}

Correspondence to: Professor Yun Chen, Department of Ultrasound, Peking University Shenzhen Hospital, 1120 Lian-Hua Road, Shenzhen, Guangdong 518036, P.R. China

E-mail: cyun126@126.com

Professor Feng Wang, Shenzhen Key Laboratory for Drug Addiction and Medication Safety, Biomedical Research Institute, Peking University Shenzhen, The Hong Kong University of Science and Technology Medical Center, 1120 Lian-Hua Road, Shenzhen, Guangdong 518036, P.R. China

E-mail: wfeng100@126.com

Key words: neurotrophin-3, neural stem cell, ultrasound, microbubbles, denervated muscle atrophy endplate degeneration. This noninvasive, efficient and targeted treatment strategy may therefore be a potential treatment for patients with denervated muscle atrophy.

\section{Introduction}

Peripheral nerve regeneration is slow and incomplete, and this leads to the development of denervated muscle atrophy, which is the main reason for hypokinesia following peripheral nerve injury (1). The clinical consequences of muscle atrophy seriously affects the quality of life of the patients. Muscle wasting is an independent index of mortality and morbidity. There is currently no reliable pharmacological treatment to prevent muscle atrophy, so effective therapies to treat muscle atrophy are required (2). Neural stem cells (NSCs) are used as a novel therapeutic agent for repairing nerve injuries $(3,4)$. NSCs are undifferentiated cells that are widely distributed in the nervous system and when transplanted into peripheral nerves, they differentiate into neurons and form functional motor endplate nerve-muscle connections with the denervated muscle (5).

Neurotrophic factors are used by nerve tissues and previous studies have demonstrated that neurotrophin-3 (NT-3) serves a regulatory role in denervated muscle atrophy (6-8). NSCs differentiate into motor neurons and form functional nerve-muscle connections, thereby increasing the number and length of regenerated axons. However, the endogenous secretion of NT-3 is low and exogenous NT-3 lacks sufficient time to accumulate due to its short half-life (9). Thus, it was hypothesized that the transfection of NT-3 into NSCs in order to produce endogenous NT-3 may adequately supply nerves with NT-3 and promote the differentiation of NSCs into motor neurons.

A previous study demonstrated that transfection mediated by ultrasound with microbubbles (MBs) with the appropriate 
parameters exhibits low toxicity and noninvasion (10). In addition, this transfection technique enhances the effect of stem cell transplantation $(11,12)$. However, the effects of using ultrasound with MBs to transfect NT-3 into NSCs remain unknown. Thus, the current study investigated the feasibility of transfecting NT-3 into NSCs using ultrasound with MBs and the subsequent transplantation of NSCs in vivo to treat denervated muscle atrophy.

\section{Materials and methods}

Animal model. A total of 32 male Sprague-Dawley rats ( $\mathrm{n}=8$ per group, 200-220 g, 8-10 weeks old) purchased from Guangdong Medical Laboratory Animal Center (Foshan, China) were used in the current study. These rats were individually housed under a constant temperature $\left(23 \pm 2^{\circ} \mathrm{C}\right)$ and maintained in a 12-h light/dark cycle with free access to food and water. Rats were anesthetized intraperitoneally with $300 \mathrm{mg} / \mathrm{kg}$ chloral hydrate (TargetMol, Boston, MA, USA) prior to transplantation of NSCs. The hair on the thighs of the rats was removed and each animal was placed in the prostrate position on an operating table. A $2 \mathrm{~cm}$ skin incision parallel to the femur and inferior at $1 \mathrm{~cm}$ was made on the right thigh of the rat to expose the sciatic nerve. The nerve was cut off at $1.5 \mathrm{~cm}$, piercing the piriformis, and a neurological defect of $1 \mathrm{~cm}$ was made. The proximal nerve was ligated using a 7-0 noninvasive micro stitch and the distal end was placed aside. The wound was washed with $0.1 \%$ povidone-iodine and sutured. All animal experiments were conducted in accordance with the guidelines developed by the National Institutes of Health (13) and approved by the Institutional Animal Care and Use Committee of Peking University Shenzhen Hospital (permit no. 09-215).

Ultrasound equipment. The ultrasound system used in the current study included an arbitrary AFG3102 waveform generator (Tektronix, Inc., Beaverton, OR, USA), an AR150A100B RF power amplifier (AR, Inc., Souderton, PA, USA) and a 1.0 MHz unfocused single-element transducer (Panametrics, Inc., Waltham, MA, USA). The parameters of ultrasonic intensity and repetition frequency were optional.

NSC culture. Hippocampi from 7 embryonic day (E)14 Sprague-Dawley rats purchased from Guangdong Medical Laboratory Animal Center (Foshan, China; permit no. SCXK2013-0002) were isolated in a biological safety cabinet and placed in a flask for primary NSC culture. Hippocampal tissues were sheared into $1 \mathrm{~mm}^{3}$ sized tissue blocks. $0.25 \%$ trypsin (EMD Millipore, Billerica, MA, USA; 1:250) was used to digest the tissue block at room temperature for $20 \mathrm{~min}$. The digested tissue solution was collected and placed in a $15 \mathrm{ml}$ centrifuge tube, and then Dulbecco's modified Eagle's medium (DMEM)/F12 (1:1 v/v, Gibco; Thermo Fisher Scientific, Inc., Waltham, MA, USA) containing fetal bovine serum (Gibco; Thermo Fisher Scientific, Inc.) was added to stop the digestion. Thereafter, the solution was centrifuged at $450 \mathrm{x} \mathrm{g}$ at room temperature for $5 \mathrm{~min}$. Then, cells were harvested and cultured in DMEM/F12 (1:1 v/v) containing 2\% B27 (Gibco; Thermo Fisher Scientific, Inc.) and 1\% N2 (Gibco; Thermo Fisher Scientific, Inc.) supplements, $0.5 \mathrm{mM}$
L-glutamine (Hyclone; GE Healthcare, Logan, UT, USA), $0.5 \mathrm{mM}$ non-essential amino acids, $20 \mathrm{ng} / \mathrm{ml}$ basic fibroblast growth factor (Promega Corp., Madison, WI, USA), 50 IU/ml penicillin (Hyclone; GE Healthcare) and $50 \mu \mathrm{g} / \mathrm{ml}$ streptomycin (Hyclone; GE Healthcare). The cells were cultured in an incubator containing $5 \% \mathrm{CO}_{2}$ at $37^{\circ} \mathrm{C}$. The culture medium was replaced half every 3 days, and the cells were passaged after 7 days of culture. Cells were observed and used at day 20 .

Transfection of NT-3. A total of $200 \mu \mathrm{MBs}\left(3 \times 10^{8} / \mathrm{ml}\right.$; Bracco Spa, Milan, Italy) with a mean size of $2-5 \mu \mathrm{m}$ were mixed with $20 \mu \mathrm{l}$ pEGFP-NT-3 recombinant plasmid $(1 \mu \mathrm{g} / \mu \mathrm{l})$ (Corning Incorporated, Corning, NY, USA) in 24-well plates at room temperature for $30 \mathrm{~min}$. The treatments used in each group were as follows: i) In the control group $500 \mu \mathrm{l} \mathrm{NSCs}\left(3 \times 10^{5} / \mathrm{ml}\right)$ were incubated in complete medium (mentioned above) with no supplements; ii) in the NSC+MB+pEGFP-NT-3 group the number of NSCs following trypsin digestion were counted (500 $\left.\mu \mathrm{l} ; 3 \times 10^{5} / \mathrm{ml}\right)$ and mixed with $20 \mu \mathrm{l}$ pEGFP-NT-3 recombinant plasmid $(1 \mu \mathrm{g} / \mu \mathrm{l})$ and $200 \mu \mathrm{l} \mathrm{MBs}$; iii) in NSC+MB group the number of NSCs following trypsin digestion were counted $\left(500 \mu \mathrm{l} ; 3 \times 10^{5} / \mathrm{ml}\right)$ and mixed with $200 \mu \mathrm{l} \mathrm{MBs}$; and iv) in the NSC+MB+NT-3 group the number of NSCs following trypsin digestion were counted $\left(500 \mu \mathrm{l} ; 3 \times 10^{5} / \mathrm{ml}\right)$ and mixed with $200 \mu \mathrm{l} \mathrm{MBs}$ and NT-3 protein (PeproTech, Inc., Rocky Hill, NJ, USA) (100 $\mu \mathrm{l} ; 50 \mathrm{ng} / \mathrm{ml})$ in place of the plasmid. The ultrasound probe was then placed below each well to sonicate the mixture for the transfection of NT-3 using the following parameters: Ultrasonic intensity, $1.5 \mathrm{~W} / \mathrm{cm}^{2}$; sonication time, $60 \mathrm{sec}$; duty cycle, $25 \%$; and MB concentration, $3 \times 10^{8} / \mathrm{ml}$. Cells were then incubated with $5 \% \mathrm{CO}_{2}$ at $37^{\circ} \mathrm{C}$ for $48 \mathrm{~h}$.

Transplantation of NSCs. NSCs were collected and centrifuged at $450 \mathrm{x} \mathrm{g}$ at room temperature for $5 \mathrm{~min}$ following sonication. A microsyringe at a depth of $4 \mathrm{~mm}$ was then used to vertically inject $100 \mu \mathrm{NSC}\left(1 \times 10^{7} / \mathrm{ml}\right)$ suspension into the upper third of the right tibialis anterior muscle in the belly of the rats $(n=8$ in each group). The needle was retained in the muscle for $10 \mathrm{~min}$ and 200,000 units penicillin was then intramuscularly injected into the left thigh.

Tibialis anterior muscle weight measurement. After being sacrificed, the bilateral tibialis anterior muscle was dissected completely from the origin to the insertion and weighed immediately with an electron scale with $0.001 \mathrm{~g}$ precision. The muscle wet weight ratio of affected-side/health-side was compared between groups to exclude the individual differences in the weight of the tibialis anterior muscle.

Tissue preparation and histological examination. All rats were sacrificed 6 weeks following transplantation for histological evaluation. The entirety of the bilateral tibialis anterior muscle with $2 \mathrm{~mm}$ deep peroneal nerve muscular branches of the anterior tibial muscle were immediately removed and samples were weighed using an analytical balance (accuracy to $0.001 \mathrm{~g}$ ). The muscle from the right thigh was then divided into four parts: The tissue block on the right side of the nerve entering point otherwise known as muscle hilus; the tissue 
block on the left side of the muscle hilus; and two tissue blocks $(1 \times 1 \times 2 \mathrm{~mm})$ around the muscle hilus.

Hematoxylin and eosin $(H \& E)$ staining. The tissue blocks on the right side of the muscle hilus were fixed in $10 \%$ buffered neutral formalin at room temperature for 12 hand a series of sections $(5 \mu \mathrm{m})$ were cut for H\&E staining at room temperature for $15 \mathrm{~min}$. Three fields of view of each section were randomly captured at a magnification of $x 200$ (Olympus BX53, Olympus Corporation, Tokyo, Japan) and analyzed using ImageJ software (version 1.48, National Institutes of Health, Bethesda, MD, USA). Four muscle fibers were randomly selected and their cross-sectional areas were measured.

Acetylcholinesterase (AChE) staining. AChE staining was used to assess the number of motor endplates in all groups. The tissue blocks on the left side of the muscle hilus were embedded in optimum cutting temperature compound for cryosection at $-20^{\circ} \mathrm{C}$ for $10 \mathrm{~min}$. The serial sections were frozen, repeatedly washed with PBS, and incubated in AChE incubation solution (Beijing Leagene Biotech Co., Ltd., Beijing, China) at $4^{\circ} \mathrm{C}$ for $12 \mathrm{~h}$ and washed again with PBS. The washed sections were then mounted with neutral gum. Three sections were randomly selected from each rat. Two fields of view of each section were randomly selected and observed using an Olympus BX53 microscope at a magnification of $x 100$. The number of motor endplates was counted.

Gold chloride staining. Gold chloride staining was used to estimate the shape and number of motor endplates. The tissue blocks around the muscle hilus (1x1x2 mm) were fixed in $20 \%$ formic acid solution at room temperature for $5 \mathrm{~h}$ and then stained with $1 \%$ gold chloride until the color changed to a brown-yellow shade at room temperature for $1 \mathrm{~h}$. The tissue blocks were then washed three times with distilled water and restored in $20 \%$ formic acid solution until the color changed to a chocolate brown. The tissue was then washed twice with distilled water and stored in glycerin until it softened. Following this, the tissue samples were mounted on glass slides. Two fields of view of each section were randomly selected and observed using an Olympus BX53 microscope at a magnification of x100.

Transmission electron microscopy (TEM). TEM was used to evaluate the ultrastructural basis of the attenuation of denervated muscle atrophy. The tissue blocks around the muscle hilus $(1 \times 1 \times 2 \mathrm{~mm})$ were fixed by perfusion using $2.5 \%$ paraformaldehyde and $1.5 \%$ glutaraldehyde in $0.1 \mathrm{M}$ phosphate buffer ( $\mathrm{pH} 7.2)$ at room temperature for $24 \mathrm{~h}$ and then post-fixed in a solution of $1 \%$ osmium tetroxide and $1.5 \%$ potassium ferrocyanine $\left(1 \mathrm{~h}, 4^{\circ} \mathrm{C}\right)$. The tissues were then dehydrated with increasing ethanol $(25,50,70,90$ and $100 \%)$ for $5 \mathrm{~min}$ each. The samples were sectioned $(50 \mathrm{~nm})$ and observed using a LIBRA 200 FE microscope (Zeiss GmbH, Jena, Germany).

Statistical analysis. Data are expressed as the mean \pm standard error of the mean. The experiments were repeated three times. One-way analysis of variance followed by Tukey's post hoc test was used to compare the means between groups using

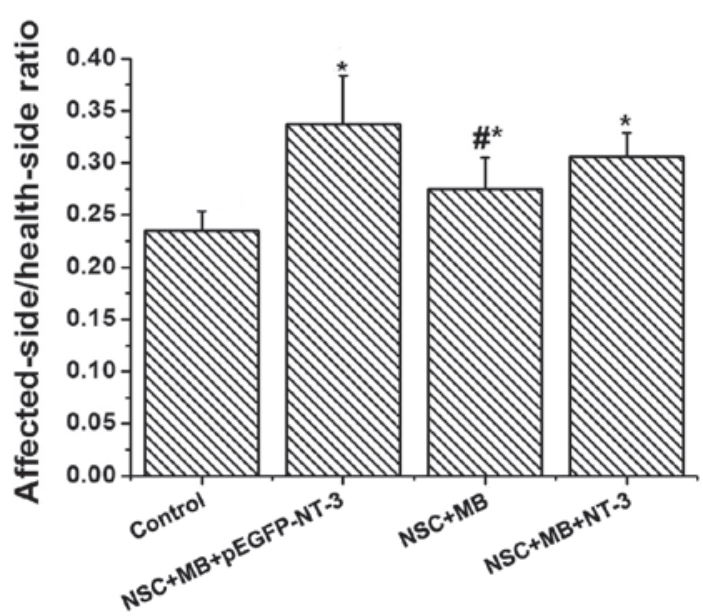

Figure 1. Comparison of tibialis anterior muscle wet weight in the control, $\mathrm{NSC}+\mathrm{MB}+$ pEGFP-NT-3, NSC+MB and NSC+MB+NT-3 groups. ${ }^{*} \mathrm{P}<0.05$ vs control; ${ }^{\#} \mathrm{P}<0.05$ vs. NSC+MB+pEGFP-NT-3. NSC, neural stem cell; MB, microbubble; NT-3, neurotrophin-3.

SPSS (version 17.0; SPSS, Inc., Chicago, IL, USA). P<0.05 was considered to indicate a statistically significant difference.

\section{Results}

Comparison of the tibialis anterior muscle wet weight between groups. The affected-side/health-side ratio of the muscle wet weight was compared between groups to exclude the individual differences in the weight of the tibialis anterior muscle (Fig. 1). The NSC+MB+pEGFP-NT-3 group had the highest ratio, whereas the control group had the lowest. The ratios in the NSC+MB+pEGFP-NT-3, NSC+MB and NSC+MB+NT-3 groups were significantly increased compared with the control $(\mathrm{P}<0.05)$. A pairwise comparison also identified that the affected-side/health-side ratio of the muscle wet weight was significantly increased in the NSC+MB+pEGFP-NT-3 group compared with the NSC+MB group $(\mathrm{P}<0.05)$.

Comparison of muscle fiber H\&E staining and cross-sectional area measurements between groups. Following $\mathrm{H} \& \mathrm{E}$ staining of the tibialis anterior muscle, visual observation of the sections demonstrated that the control group had the smallest cross-sectional area of muscle fibers, followed by the NSC+MB and NSC+MB+NT-3 groups, while the $\mathrm{NSC}+\mathrm{MB}+\mathrm{pEGFP}-\mathrm{NT}-3$ group had the largest cross-sectional area (Fig. 2). The comparison made using ImageJ software revealed significant differences in the cross-sectional area of the muscle fiber among the groups (Fig. 3). Pairwise comparison demonstrated that the cross sectional area of the muscle fibers in the NSC+MB+pEGFP-NT-3, NSC+MB and NSC+MB+NT-3 groups was significantly increased compared with the control $(\mathrm{P}<0.05)$. In addition, the cross sectional area of the muscles fibers in the NSC+MB and NSC+MB+NT-3 groups was significantly decreased compared with the NSC+MB+pEGFP-NT-3 group $(\mathrm{P}<0.05)$. However, the difference in the cross-sectional area of the muscle fibers between the NSC+MB and NSC+MB+NT-3 groups was not significant. 

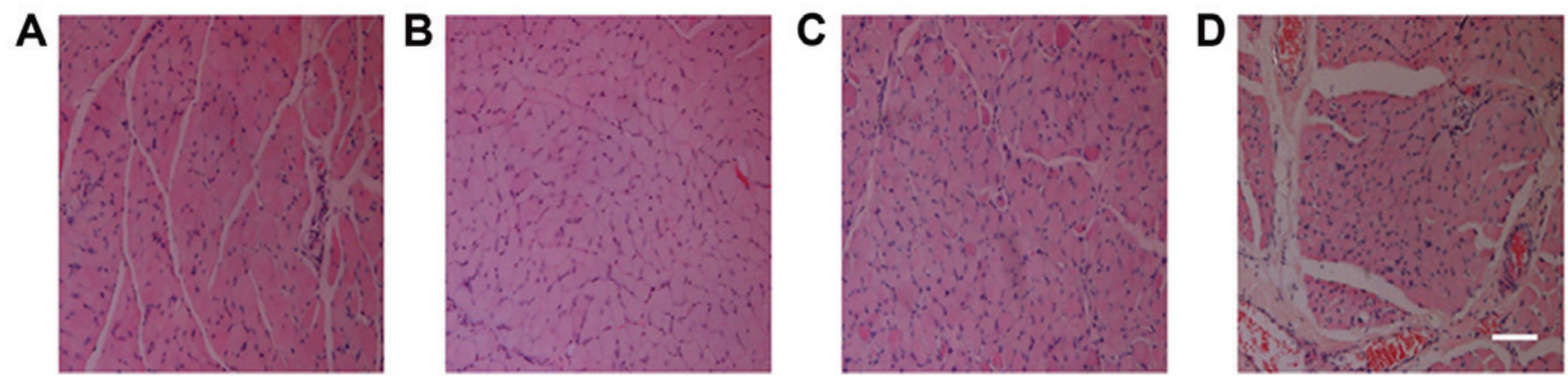

Figure 2. H\&E staining of the tibialis anterior muscle demonstrating the cross-section of muscle fibers. H\&E staining of the (A) control, (B) NSC+MB+pEGFP-NT-3, (C) NSC+MB and (D) NSC+MB+NT-3 groups. Scale bar, 20 mm. Magnification, x200. H\&E, hematoxylin and eosin; NSC, neural stem cell; $\mathrm{MB}$, microbubble; NT-3, neurotrophin-3.

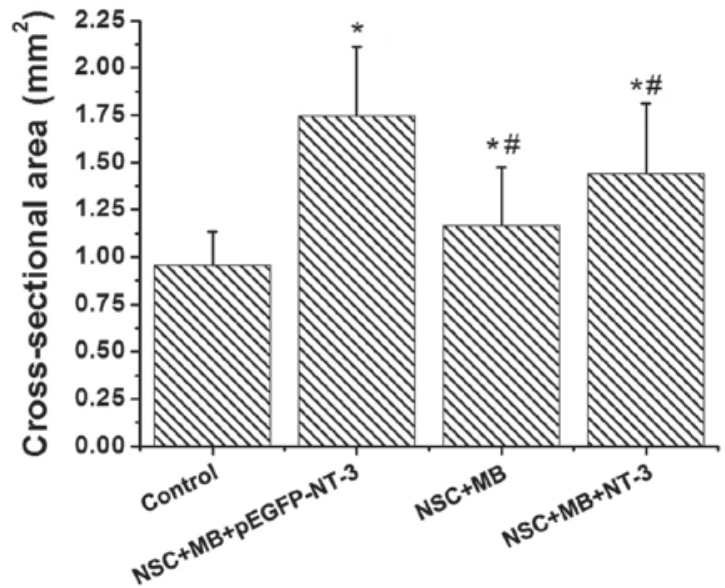

Figure 3. Analysis of tibialis anterior muscle fiber cross-sections stained 1with $\mathrm{H} \& \mathrm{E}$. Analysis of H\&E staining in the control, NSC+MB+pEGFP-NT-3, $\mathrm{NSC}+\mathrm{MB}$ and $\mathrm{NSC}+\mathrm{MB}+\mathrm{NT}-3$ groups. ${ }^{*} \mathrm{P}<0.05$ vs. control; ${ }^{*} \mathrm{P}<0.05$ vs. $\mathrm{NSC}+\mathrm{MB}+$ pEGFP-NT-3. H\&E, hematoxylin and eosin; NSC, neural stem cell; MB, microbubble; NT-3, neurotrophin-3.

Comparison of AChE staining between groups. AChE staining was used to determine the number of motor endplates in each group (Fig. 4). The lowest number was recorded in the control. Pairwise comparison demonstrated that the number of motor endplates in the NSC+MB+pEGFP-NT-3 group was significantly increased compared with the NSC+MB group, and the number in the control was significantly decreased compared with the NSC+MB and NSC+MB+NT-3 groups (all $\mathrm{P}<0.05$; Fig. 5). However, the difference in the number of motor endplates between the NSC+MB and NSC+MB+NT-3 groups was not significantly different (Fig. 5).

Comparison of gold chloride staining between groups. Normal (control group) motor endplates stained with gold chloride exhibited a horseshoe or oval shape and clear color, whereas normal muscle fibers were irregularly shaped (Fig. 6). The motor endplates in the experimental groups (NSC+MB and NSC+MB+NT-3 groups) were fewer, smaller, unevenly dyed and irregular. In addition, atrophy manifestations and fibrous connective tissue increased to different levels. The motor endplates in the control, NSC+MB and $\mathrm{NSC}+\mathrm{MB}+\mathrm{NT}-3$ groups exhibited unclearly stained areas with an irregular shape and were markedly decreased compared with the NSC+MB+pEGFP-NT-3 group, in which motor endplates exhibited a more regular shape.

Comparison of TEM results between groups. The muscle fibers in the NSC+MB+pEGFP-NT-3 group differed slightly compared with the normal (control group) ultrastructure (Fig. 7). The $\mathrm{Z}$ lines, dark band and $\mathrm{M}$ line were clear, and their shapes were normal. In addition, muscle fiber atrophy and a slightly loose arrangement were observed, and the mitochondrial cristas were shorter. In the NSC+MB+NT-3 and NSC+MB groups, the $\mathrm{Z}$ line was slightly blurred, the dark bands and $M$ lines were not clear, the muscle fibers were not neatly arranged. In the muscle fibers of the control group severely abnormal morphology was observed, the Z line was blurred and discontinuous, and the dark bands and $\mathrm{M}$ lines were unclear. In the NSC+MB and MSC+MB+NT-3 groups, the motor endplate synaptic cleft was shortened and the secondary folds became shallow or disappeared (Fig. 8). In the NSC+MB+pEGFP-NT-3 group, the secondary folds became slightly shallow and the number of synaptic vesicles in the motor endplates was increased. There were no clear motor endplates present in the control group.

\section{Discussion}

Preventing the atrophy of denervated muscle following peripheral nerve injury is challenging; however, it has been demonstrated that stem cell transplantation may be used as a treatment (14). Kubo et al (5) demonstrated that the co-incubation of myotubules and motor neurons differentiated from NSCs lead to new nerve-muscle connections forming in vitro. In addition, it has been demonstrated using animal experiments that local intramuscular injection of motor neurons may relieve muscle denervation atrophy (4). The study demonstrated that locally transplanted NSCs are able to survive in the muscle and form new nerve-muscle connections to attenuate denervated muscle atrophy (4).

NT-3 is an early signaling factor that induces NSC differentiation by inhibiting the basic fibroblast growth factor during the proliferation of embryonic NSCs. Lim et al (7) conducted an experiment on mice, which demonstrated that by binding to neurotrophic tyrosine kinase receptor type 3 on the cell membrane, NT-3 amplifies the 
A

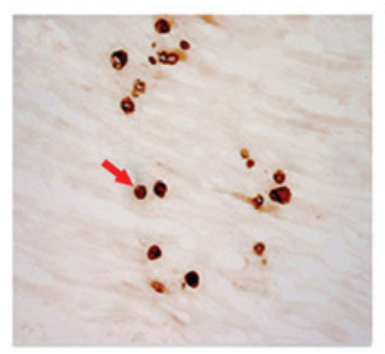

B

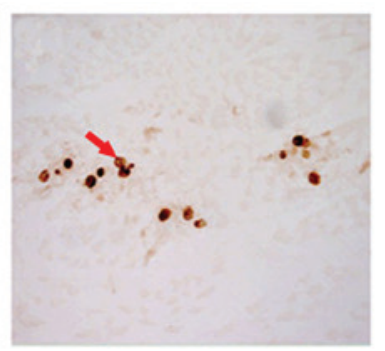

C

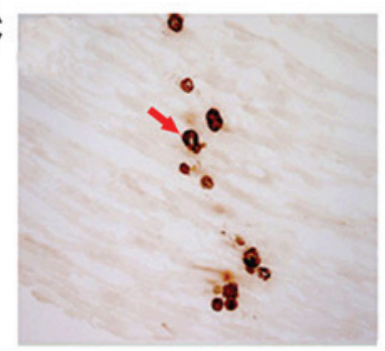

D

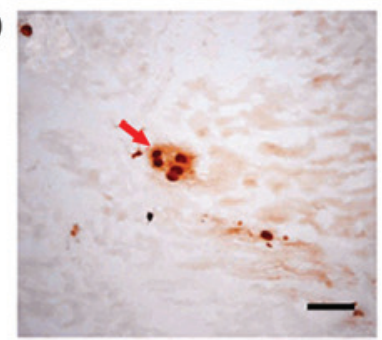

Figure 4. Motor endplates stained with acetylcholinesterase. Acetylcholinesterase staining of motor endplates in the (A) control, (B) NSC+MB+pEGFP-NT-3, (C) NSC+MB and (D) NSC+MB+NT-3 groups. Arrows indicate motor endplates. Scale bar, 10 mm. Magnification, x100. NSC, neural stem cell; MB, microbubble; NT-3, neurotrophin-3.

phosphoinositide-3-kinase/protein kinase B and extracellular signal-regulated kinase signaling pathways to induce NSC differentiation into neurons, and induces synaptic growth by the specific phosphorylation of mitogen-activated protein kinase 14. It was also demonstrated that NT-3 sustains the survival of muscle spindles, tendons and skin afferent sensory neurons, promotes motor neuron regeneration and nerve-muscle connection maturation, and increases the number and length of regenerative axons and NSCs, thereby preventing muscle atrophy following nerve injury. NT-3 has a continuous and long-term effect on muscle cells, but exogenous neurotrophic factors have low biological activity, limited sources and a short half-life, which restricts their function (9).

The current focus of research into treatments for denervated muscle atrophy is on resolving the shortcomings of NT-3 and its application. It has previously been demonstrated that gene transfer is a reliable method of inducing stable and sustained expression of NT-3 in vivo (15). Common methods of transfection include viral and liposomal transfection. However, the biosecurity of viral transfection poses a challenge to its clinical application (16). During liposomal transfection cationic liposomes may move from the serum and accumulate in the lung tissues, inducing a strong anti-inflammatory response, thus limiting its clinical application $(17,18)$. Micron-sized MB contrast agents for ultrasonography have been widely used in clinical settings $(19,20)$. Ultrasound with MBs is a recognized technology for enhancing the efficiency of transfection (21). It has been considered that the mechanism by which ultrasound with MBs enhances transfection is based on the cavitation effect, where at a certain ultrasound frequency, the MBs vibrate rapidly and collapse into pieces, producing shock waves, jet streams and other strong localized effects that increase cell membrane permeability and gene delivery (22). Ultrasound with MBs significantly enhances the efficiency of transfection and expression of the gene transfected, due to its low toxicity and immunogenicity. Thus, ultrasound with MBs has become the primary focus of numerous studies worldwide.

To the best of our knowledge, NSC transfection using ultrasound with MBs was applied for the first time in the present study. At 6 weeks following nerve injury, the rats in the NSC+MB+pEGFP-NT-3 group exhibited the highest affected-side/health-side ratio of muscle wet weight in the cross-sectional area. Furthermore, TEM demonstrated that the muscle fibers in the NSC+MB+pEGFP-NT-3 group differed compared with the control, and the $\mathrm{Z}$

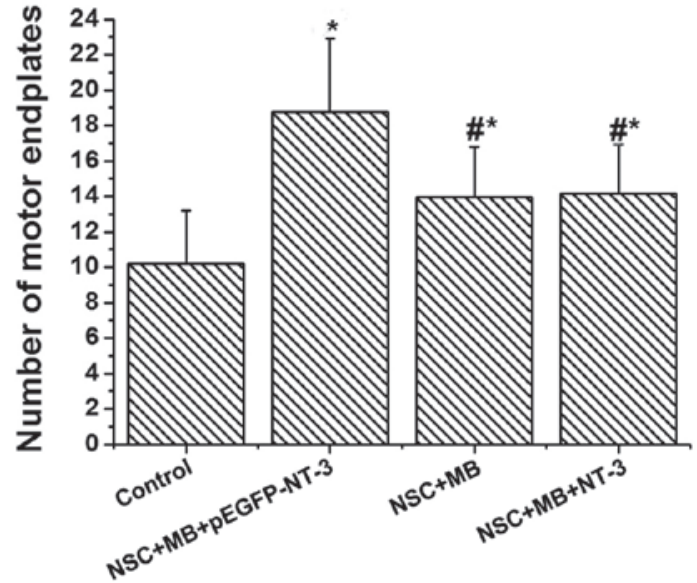

Figure 5. Analysis of the number of motor endplates stained with acetylcholinesterase in the control, NSC+MB+pEGFP-NT-3, NSC+MB and $\mathrm{NSC}+\mathrm{MB}+\mathrm{NT}-3$ groups. ${ }^{*} \mathrm{P}<0.05$ vs. control; ${ }^{\#} \mathrm{P}<0.05$ vs. NSC+MB+pEGFP-NT-3. NSC, neural stem cell; MB, microbubble; NT-3, neurotrophin-3.

lines, dark band and $\mathrm{M}$ line were clear, and their shapes were normal. NSC+MB+pEGFP-NT-3 group could better maintain muscle weight compared with other groups, and had the heaviest muscle fibers among all groups. TEM further revealed differences in the motor endplates of the $\mathrm{NSC}+\mathrm{MB}+\mathrm{pEGFP}-\mathrm{NT}-3$ group, and gold chloride staining demonstrated that an increased number of motor endplates in the NSC+MB+pEGFP-NT-3 group retained regular shapes compared with the other three groups. AChE staining demonstrated that the NSC+MB+pEGFP-NT-3 group had the highest number of motor endplates among the groups. These results suggest that the NSC+MB+pEGFP-NT-3 group had the lowest rate of motor endplate degeneration, and that the transfection of NT-3 into NSCs using ultrasound with MBs and the subsequent transplantation of NSCs in vivo attenuates denervated muscle atrophy. The difference of histological and electron microscopic observation between the NSC+MB and $\mathrm{MSC}+\mathrm{MB}+\mathrm{NT}-3$ groups was not marked, possibly due to the NT-3 protein being metabolized in the MSC+MB+NT-3 group, which limited it from exerting stable neurotrophic effects.

In conclusion, the results of the present study suggest that the transfection of NT-3 into NSCs using ultrasound with MBs, and the subsequent transplantation of the cells 
A

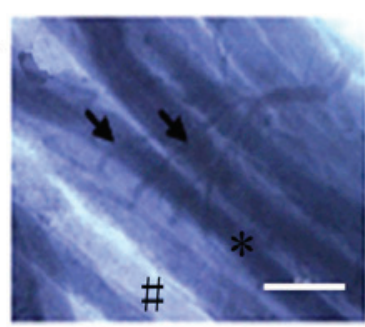

B

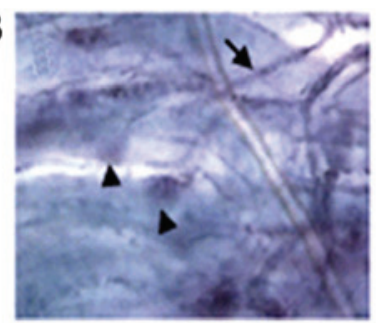

C

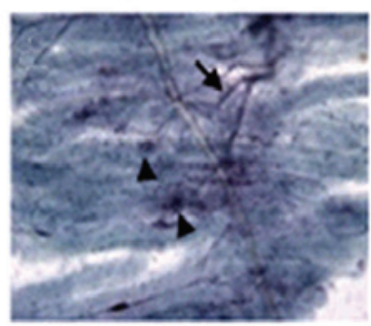

D

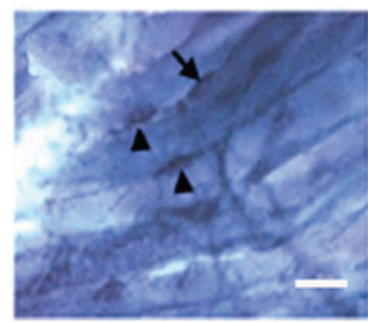

Figure 6. Motor endplate and muscle fibers stained with gold chloride. Gold chloride staining in the (A) control, (B) NSC+MB+pEGFP-NT-3, (C) NSC+MB and (D) NSC+MB+NT-3 groups. Arrows indicate nerve fibers, arrowheads indicate motor endplates, "indicates atrophy manifestation and ${ }^{\#}$ indicates fibrous connective tissues. Scale bar, $50 \mu \mathrm{m}$. NSC, neural stem cell; MB, microbubble; NT-3, neurotrophin-3.

A

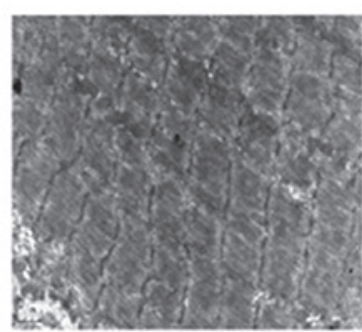

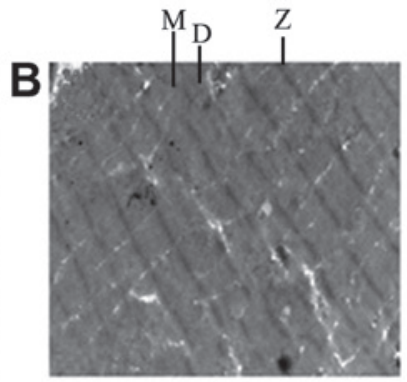
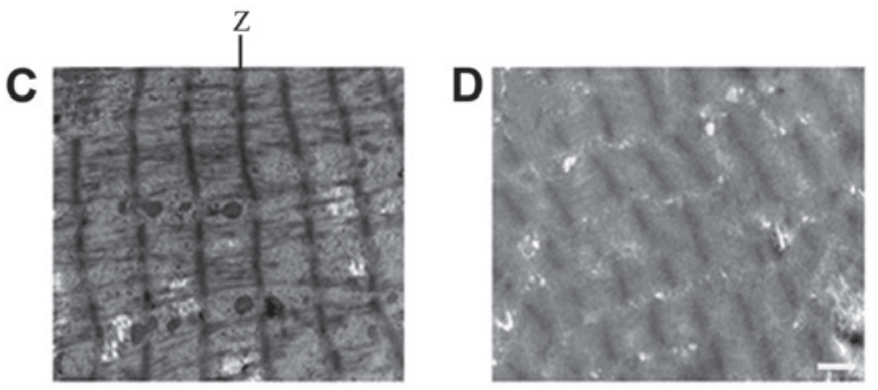

Figure 7. TEM images of muscle fibers. TEM images of the (A) control, (B) NSC+MB+pEGFP-NT-3, (C) NSC+MB and (D) NSC+MB+NT-3 groups. Scale bar, $1 \mu \mathrm{m}$. TEM, transmission electron microscopy; NSC, neural stem cell; MB, microbubble; NT-3, neurotrophin-3; Z, Z line; M, M line; D, dark bands.
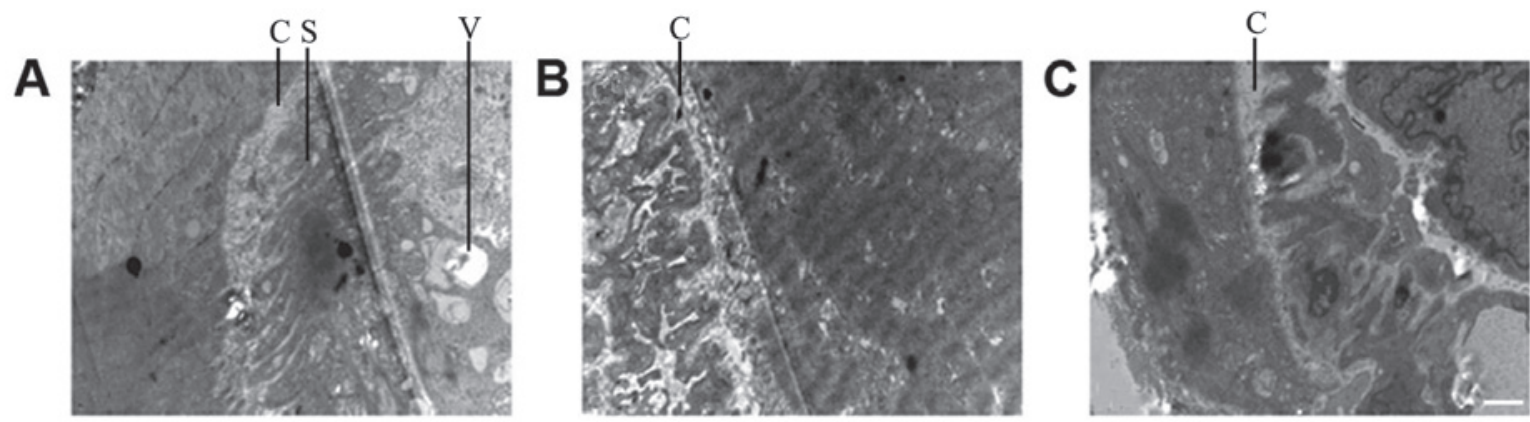

Figure 8. TEM images of motor endplates. TEM images of the (A) NSC+MB+pEGFP-NT-3, (B) NSC+MB and (C) NSC+MB+NT-3 groups. Scale bar, $1 \mu \mathrm{m}$. TEM, transmission electron microscopy; NSC, neural stem cell; MB, microbubble; NT-3, neurotrophin-3; C, synaptic cleft; S, secondary folds; V, synaptic vesicles.

in vivo, is a novel, safe and effective strategy for preventing and treating denervated muscle atrophy and promoting the functional recovery of peripheral nerves in clinical settings. The future development of novel types of MBs is required to improve the efficiency of transfection. This development may reduce the morbidity of patients with peripheral nerve injury, thereby providing social and economic benefits.

\section{Acknowledgements}

The present study was supported by the National Natural Science Foundation of China (grant no. U1204810), Guangdong Natural Science Foundation (grant nos. 2014A030313709, 2014A030313710 and 2015A030313889), Shenzhen
Science and Technology Planning Project (grant nos. ZDSYS201504301045406, JCYJ20170413100222613, JCYJ20170306154931588，JCYJ20150403110829621, JCYJ20140415162338855，JCYJ20140415162542975 JCYJ20140415162338774 and JCYJ20140828163634004) and the Guangdong Bureau of Traditional Chinese Medicine Project (grant no. 20171228).

\section{References}

1. Armstrong RJ, Harrower TP, Hurelbrink CB, McLaughin M, Ratcliffe EL, Tyers P, Richards A, Dunnett SB, Rosser AE and Barker RA: Porcine neural xenografts in the immunocompetent rat: Immune response following grafting of expanded neural precursor cells. Neuroscience 106: 201-216, 2001. 
2. Su Z, Hu L, Cheng J, Klein JD, Hassounah F, Cai H, Li M, Wang $\mathrm{H}$ and Wang $\mathrm{XH}$ : Acupuncture plus low-frequency electrical stimulation (Acu-LFES) attenuates denervation-induced muscle atrophy. J Appl Physiol (1985) 120: 426-436, 2016.

3. Bost F, Caron L, Marchetti I, Dani C, Le Marchand-Brustel Y and Binétruy B: Retinoic acid activation of the ERK pathway is required for embryonic stem cell commitment into the adipocyte lineage. Biochem J 361: 621-627, 2002.

4. Dooley D, Vidal P and Hendrix S: Immunopharmacological intervention for successful neural stem cell therapy: New perspectives in CNS neurogenesis and repair. Pharmacol Ther 141: 21-31, 2014.

5. Kubo T, Randolph MA, Groger A and Winograd JM: Embryonic stem cell-derived motor neurons form neuromuscular junctions in vitro and enhance motor functional recovery in vivo. Plast Reconstr Surg 123 (2 Suppl): 139S-148S, 2009.

6. Lawrie A, Brisken AF, Francis SE, Cumberland DC, Crossman DC and Newman CM: Microbubble-enhanced ultrasound for vascular gene delivery. Gene Ther 7: 2023-2027, 2000

7. Lim MS, Nam SH, Kim SJ, Kang SY, Lee YS and Kang KS: Signaling pathways of the early differentiation of neural stem cells by neurotrophin-3. Biochem Biophys Res Commun 357: 903-909, 2007.

8. Lin S, Xu J, Hu S, Xu L, Zhang C, Wang Y and Gu Y: Combined application of neutrophin-3 gene and neural stem cells is ameliorative to delay of denervated skeletal muscular atrophy after tibial nerve transection in rats. Cell Transplant 20: 381-390, 2011

9. Sahenk Z, Galloway G, Clark KR, Malik V, Rodino-Klapac LR, Kaspar BK, Chen L, Braganza C, Montgomery C and Mendell JR AAV1.NT-3 gene therapy for charcot-marie-tooth neuropathy. Mol Ther 22: 511-521, 2014.

10. Raju BI, Leyvi E, Seip R, Sethuraman S, Luo X, Bird A, Li S and Koeberl D: Enhanced gene expression of systemically administered plasmid DNA in the liver with therapeutic ultrasound and microbubbles. IEEE Trans Ultrason Ferroelectr Freq Control 60 88-96, 2013

11. Lin WH, Fan CH, Ting CY, Liu HL and Yeh CK: Dynamic perfusion assessment by contrast-enhanced ultrasound in blood-brain barrier disruption. Conf Proc IEEE Eng Med Biol Soc 2013: $1152-1155,2013$.

12. Ménard C, Hein P, Paquin A, Savelson A, Yang XM, Lederfein D, Barnabé-Heider F, Mir AA, Sterneck E, Peterson AC, et al: An essential role for a MEK-C/EBP pathway during growth factor-regulated cortical neurogenesis. Neuron 36: 597-610, 2002.
13. Luo YX, Xue YX, Liu JF, Shi HS, Jian M, Han Y, Zhu WL, Bao YP, Wu P, Ding ZB, et al: A novel UCS memory retrieval-extinction procedure to inhibit relapse to drug seeking. Nat Commun 6: 7675, 2015.

14. Modo M, Rezaie P, Heuschling P, Patel S, Male DK and Hodges H: Transplantation of neural stem cells in a rat model of stroke: Assessment of short-term graft survival and acute host immunological response. Brain Res 958: 70-82, 2002.

15. Nomikou N, Feichtinger GA, Redl H and McHale AP: Ultrasound-mediated gene transfer (sonoporation) in fibrin-based matrices: Potential for use in tissue regeneration. J Tissue Eng Regen Med 10: 29-39, 2016.

16. Fan Z, Kumon RE and Deng CX: Mechanisms of microbubble-facilitated sonoporation for drug and gene delivery. Ther Deliv 5: 467-486, 2014.

17. Nomikou N and McHale AP: Exploiting ultrasound-mediated effects in delivering targeted, site-specific cancer therapy. Cancer Lett 296: 133-143, 2010.

18. Ochi M, Kwong WH, Kimori K, Takemoto S, Chow SP and Ikuta Y: Delay of the denervation process in skeletal muscle by sensory ganglion graft and its clinical application. Plast Reconstr Surg 97: 577-586, 1996.

19. Phillips LC, Klibanov AL, Wamhoff BR and Hossack JA Targeted gene transfection from microbubbles into vascular smooth muscle cells using focused, ultrasound-mediated delivery. Ultrasound Med Biol 36: 1470-1480, 2010.

20. Shih R, Bardin D, Martz TD, Sheeran PS, Dayton PA and Lee AP: Flow-focusing regimes for accelerated production of monodisperse drug-loadable microbubbles toward clinical-scale applications. Lab Chip 13: 4816-4826, 2013.

21. Wu J and Nyborg WL: Ultrasound, cavitation bubbles and their interaction with cells. Adv Drug Deliv Rev 60: 1103-1116, 2008.

22. Yohn DC, Miles GB, Rafuse VF and Brownstone RM: Transplanted mouse embryonic stem-cell-derived motoneurons form functional motor units and reduce muscle atrophy. J Neurosci 28: 12409-12418, 2008.

This work is licensed under a Creative Commons

Attribution-NonCommercial-NoDerivatives 4.0 International (CC BY-NC-ND 4.0) License. 